\title{
Perceiving space and optical cues via a visuo-tactile sensory substitution system: a methodological approach for training of blind subjects for navigation
}

\author{
Hervé Segond ${ }^{1}$, Déborah Weiss ${ }^{1}$, Magdalena Kawalec ${ }^{2}$, Eliana Sampaio ${ }^{3}$ \\ ${ }^{1}$ Faculté de Psychologie, Laboratoire de Psychologie des Cognitions EA 4440, Université de \\ Strasbourg (UdS), 12 rue Goethe, 67000 Strasbourg, France; e-mail: herve.segond@unistra.fr; \\ ${ }^{2}$ Centre National de la Recherche Scientifique (CNRS), UPR 9022, 15 rue René Descartes, \\ 67000 Strasbourg, France; ${ }^{3}$ Conservatoire National des Arts et Métiers (CNAM), 2 rue Conté, \\ 75003 Paris, France \\ Received 24 November 2008, in revised form 26 April 2013
}

\begin{abstract}
A methodological approach to perceptual learning was used to allow both early blind subjects (experimental group) and blindfolded sighted subjects (control group) to experience optical information and spatial phenomena, on the basis of visuo-tactile information transmitted by a 64-taxel pneumatic sensory substitution device. The learning process allowed the subjects to develop abilities in spatial localisation, shape recognition (with generalisation to different points of view), and monocular depth cue interpretation. During the training phase, early blind people initially experienced more difficulties than blindfolded sighted subjects (having previous perceptual experience of perspective) with interpreting and using monocular depth cues. The amelioration of the performance for all blind subjects during training sessions and the quite similar level of performance reached by two groups in the final navigation tasks suggested that early blind people were able to develop and apply cognitive understanding of depth cues. Both groups showed generalisation of the learning from the initial phases to cue identification in the maze, and subjectively experienced shapes facing them. Subjects' performance depended not only on their perceptual experience but also on their previous spatial competencies.
\end{abstract}

Keywords: sensory substitution, blindness, plasticity, depth perception, navigation, perceptual learning

\section{Introduction}

Vision is, very early in life, the preferential perceptual mode for discovering, understanding, and adapting to our environment. From birth, babies establish early coordination between vision and other perceptual modalities (Streri and Gentaz 2003). Around 3-4 months of age, perceived visual information about the distant, extra-corporeal environment initiates and controls motor activity (Hatwell 2003). The early intermodal transfers from vision to touch allow the construction of a sensorimotor visual space, improve the tactile modality thanks to visuo-tactile coordination, give a better structure to manual exploration modes of objects, and limit the difficulties for representing tactile spatial data. Thus, the cognitive consequences of visual impairment depend upon the age at which it appears. Those blinded later in life do not show strong cognitive troubles related to space recognition due to the permanent benefits of early visuo-tactile intermodal transfers. Thus, studies on the consequences of visual impairment on cognitive functions always make the distinction between early and late blindness (before 6 months or 1 year of age versus after 3 years, respectively) and compare the performance between early blind and blindfolded sighted individuals (Hatwell 2003).

Blind people develop compensatory strategies resulting in more efficient tactile (eg Braille reading) or auditory (eg localisation) spatial information processing and greater capacities of selective attention (eg Grant et al 2000). This phenomenon is correlated with cerebral plasticity (eg Hamilton and Pascual-Leone 1998) and is strongly associated with training and being actively implicated in the explored space. 
The construction of spatial representations constitutes a fundamental aspect of blind people's cognitive functioning. The main question in this domain is to determine if these non-visual spatial representations have the same functional properties as the visual images of sighted people.

In the absence of care programmes for early blind people, various perceptual and cognitive disorders appear, rising to serious pathologies in motor development, language acquisition, spatial representation, personality development, interaction with the physical and social environment (parent-child), autonomy, and self-confidence. Thus, autistic-like disorders appear more frequently within this population; about $25 \%-50 \%$ of blind children exhibit these symptoms (Sampaio 1989), compared to $0.09 \%$ in the general population of children (cf French interministry circular of 8 March $2005^{(1)}$ ). Such phenomena underline the need to develop studies on sensory substitution allowing young blind people to detect the presence and understand the nature of distant objects, and to discover the perceptual consequences of their own actions. This is crucial to the development of a feeling of effectiveness and self-confidence.

The development of sensory prostheses is based on the remarkable flexibility of the functional capacities of the perceptual systems and on cerebral plasticity - the adaptive capability of the central nervous system (Bach-y-Rita 1972, 1988, 1995). As Bach-y-Rita stated in 1972, we don't see with our eyes but with our brain. Indeed, visual perception refers to any perception dealing with visual information.

Touch is the best replacement modality for failing vision (Segond 2008), as the most redundant and intensively used by blind people to allow access to space and physical knowledge of the environment. Tactile vision substitution systems (TVSSs; see Kaczmarek and Bach-yRita 1995) are advantageous for the individual with regards to the kind of information they allow access to. TVSSs replace somesthetically the information usually conveyed by vision. Transmitting a projective 2-D tactile image of one or several 3-D objects, they allow remote tactile perception. Thus, TVSSs do not deprive one of one's auditory environments.

TVSSs collect visual information from a microvideo camera and transform it into "tactile images". These tactile stimulation patterns, which produce a feeling of pressure or vibration, are transmitted to a matrix of tactors placed on the skin of the abdomen or in contact with the tongue. TVSSs represent a tool providing information not only about location of objects in space, but also about the structure of the objects (Bach-y-Rita et al 2003).

The importance of movement for perception is essential in ecological psychology (Gibson 1966, 1979), considering the existence of a system functionally integrating sensory and motor units. More recently, Lenay et al (2000) stated that the recognition of perceived objects is based on the detection of perceptual motor invariants. This indicates the mental construction of constant connections between action and feeling. Thus, spatial location, as well as shape recognition, correspond to temporal syntheses of successive feelings, according to the rule connecting action with feeling. It is no wonder, then, that previous TVSS studies revealed the importance of the explorative movements (backwards and inversely, side movements, zoom utilisation, etc) carried out by the individual handling the camera (Bach-y-Rita 1972; Bach-y-Rita and Hughes 1985; Guarniero 1977; Miletic et al 1988).

The subjective experience resulting from TVSS's stimulation was shown to be comparable with that of vision (Bach-y-Rita 1972; Sampaio et al 2001; Segond et al 2005). Indeed, the subjects' subjective reports revealed that, at the beginning, the stimulation could be felt only through the skin; but after training, the subjects perceived 3-D objects located in extra-corporeal space in front of them without tactilely feeling them (Auvray et al 2005;

(1) Circular DGAS/DGS/DHOS/3C/2005/124 signed by the Ministry of National Education, Higher Education and Research, and the Ministry of Solidarities, Health, and Family, available at http:/www. cra-rhone-alpes.org/spip.php?article144 
Guarniero 1974; Sampaio 1995). This reflects the phenomenon of setting percepts in externality, which characterises tactile vision sensory substitution. These results call into question the traditional opposition between tactile and remote visual perception. As Proust (1997) suggested, if blind subjects receive the same information as the one inducing vision in sighted subjects, then we can consider that the same invariant properties of objects are extracted by normal and "tactile" vision.

Preliminary studies on visuo-tactile sensory substitution in the 1970s were initially motivated by the desire to provide visual aids for navigation to blind subjects (Bach-y-Rita 1972). Spatial navigation is defined by moving oneself throughout the environment using spatial information. This is a fundamental perceptual-cognitive ability usually studied within the framework of maze exploration (for review see Van Horn et al 1998). There was only one study of usage of TVSSs in navigation tasks (Jansson 1983). Thus, in our previous work (Segond et al 2005) we gave blindfolded sighted people a navigation task through the physical world, exclusively based on visuo-tactile substitution information, to test the training procedure that allowed its completion (Segond et al 2005). The previously mentioned study of Jansson (1983) concerned only one blind subject using a matrix of $12 \times 18$ vibrators on the forehead (electrophthalm system) in a very simple environment. The task was a tactually guided slalom walk towards the target pole on the sole basis of obstacle detection (vertical bars) after preliminary training. Even for this simple task, some collisions were still observed (Jansson 1983). Compared to that, our procedure of training (necessary in adults for detection of perceptual motor invariants and discrimination of geometrical shapes with the TVSS camera) and the level of performance observed in the final tasks of navigation constituted a large improvement (Segond et al 2005). We created a complex navigation task in a 3-D maze in which people (using a 96 microelectrode TVSS-matrix placed on the abdomen) had to find the way out on the basis of a perceptual analysis of directional cues, optical information about depth, and to generalise these cues as recognised from different viewpoints (ie perspective; Segond et al 2005).

For the exploration task, we used a truly 3-D environment (Segond et al 2005), rather than the virtual maze already used in other studies (Held and Durlach 1992 and Witmer and Singer 1994; quoted by Maguire et al 1999), to evaluate human navigation competence and the emerging feeling of presence (subjective experience of being somewhere while being physically elsewhere). We wanted to create a useful background allowing blind people to navigate in their real 3-D environment, instead of asking them to deal with a 2-D representation of depth in the virtual reality. To date, the ETV4 version of the TVSS, ${ }^{(2)}$ used in our previous study (Segond et al 2005), has not allowed high mobility due to the relatively short connections between the stimulating matrix and the computer interface. Also, the volume and the energy consumption of this prototype were problematic. Consequently, we used a navigation task in which the subjects had to radio-control, by manipulating two joysticks, a robot equipped with a camera and placed inside the maze. Subjects could perceive sensory and perceptual consequences of their actions through the TVSS. Thereby, a sensorimotor coupling could occur between the matrix's tactile information and visual cues located in the maze.

Sensory substitution constitutes, for blind people, the only access to this kind of information located in distant extra-corporeal space. Blindfolded sighted subjects mainly use their visual knowledge about environment to process spatial information, whereas blind people have to build the cognitive meaning of the visual-like information that the TVSS allows them to access for the first time in their life (ie perspective, depth cues, interposition, and parallax of movement). Even if blind people can express certain knowledge about optical cues, such as perspective, they do not know what it really means. Indeed, Heller and

(2) http://www.forethoughtdevelopment.com/videotac.html 
collaborators $(1996,2002)$ showed that congenitally blind people did not spontaneously understand drawings that represent perspective, nor produce foreshortened drawings, but could readily learn their meaning.

Taking all above into account, the first aim of the present study was to replicate the previous results for blindfolded sighted subjects control group even with a lower tactors density (64 tactors for the pneumatically driven tactile display-PTD). Our second aim was to implement a training procedure that could allow blind subjects to carry out the navigation task as efficiently as the blindfolded sighted subjects control group, taking into account individual differences and the unknown meaning of visual-like information. The training procedure had to allow the two groups of adults to run a successful navigation in a 3-D space following only the substitutive information provided by tactile vision. Both groups had to adapt to a new exploration mode for distant objects and space. Thus, we considered there to be no initial advantage for one group over the other. Thanks to our training procedure, we expected the success of both groups in carrying out the navigation task. Nevertheless, difficulties and individual differences were supposed to more likely appear among early blinds due to the lack of experience with visual-like information.

\section{Method}

\subsection{Subjects}

Thirteen subjects took part in the experiment: six early blind male subjects and seven blindfolded sighted subjects, three males and four females, ranging in age from 31 to 50 years for the blind $(M=41$ years, $\mathrm{SD}=6$ years) and from 24 to 50 years for the sighted $(M=34$ years, $\mathrm{SD}=8$ years). All subjects received a medical visit of the Clinical Investigation Center of the University Hospital of Strasbourg and were cleared of medical, cognitive, or motor problems. All blind subjects were either congenitally blind or lost their sight in the first weeks of life. They didn't have any light perception or residual vision (see dates of onset of blindness and causes in table 1). They lived in an independent way in the city of Strasbourg (France) and were professionally and/or socially active.

Table 1. Etiology and date of onset of blindness for the experimental group of six early blind people.

\begin{tabular}{lcl}
\hline Blind subjects & Onset of blindness/years & Etiology \\
\hline S1A & $<1$ & meningitis \\
S2A & 0 & retrolental fibroplasia \\
S3A & 0 & retrolental fibroplasia \\
S4A & 0 & bilateral optic nerve damage \\
S5A & 0 & retrolental fibroplasia \\
S6A & $<3$ & unknown (left eye enucleation) \\
\hline
\end{tabular}

\subsection{The tactile vision substitution system (TVSS)}

The visuo-tactile device used for the training was the PTD version of the TVSS. The PTD prototype has been developed and constructed by the Electronic Vision Group at the Kirchoff Institute for Physics of the University of Heidelberg along with the dedicated Visor software (V2.0) working in PC environment (Zappe et al 2004). This PTD is a nonmagnetic device specially designed to be used for both behavioural and anatomo-functional studies ran in the high magnetic field of an fMRI environment (so that it does not disturb the image acquisition). The PTD is made up of five following elements (see figure 1). The Visor software can receive images from a webcam (Philips ToUcam XS) and allows one to generate patterns by a paradigm script or interactively on the PC. The PTD control involves also the trigger, the input/output transforming unit (TIOTU), and the electronic pneumatic interface (EPI) with 


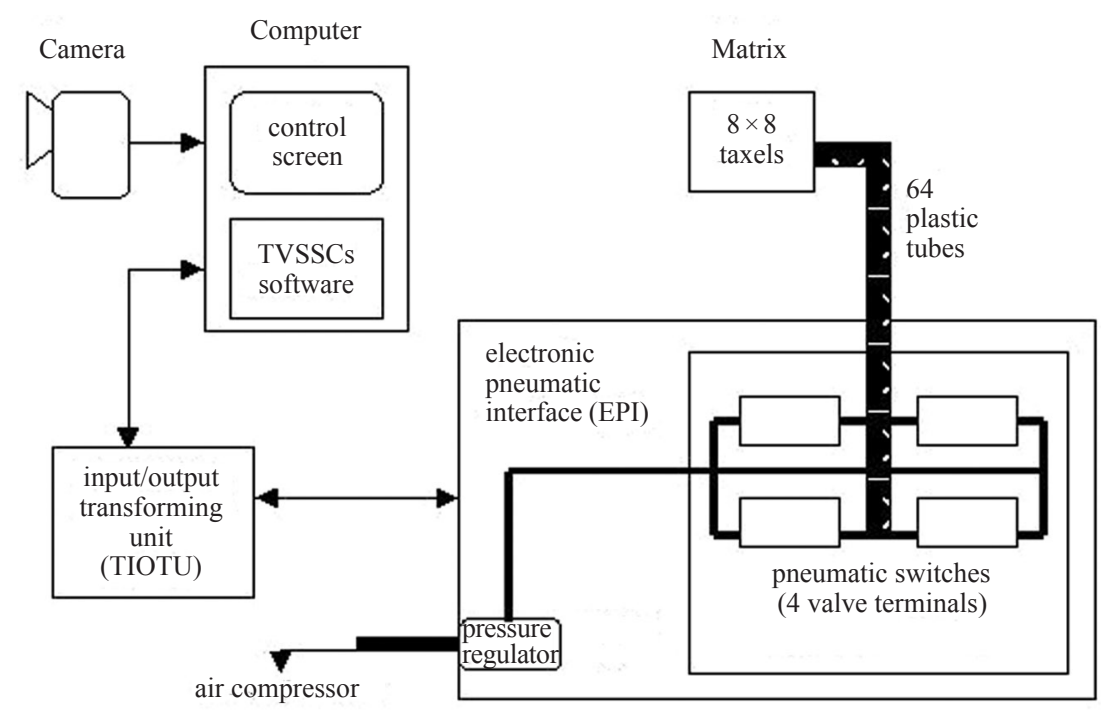

Figure 1. Diagram of the pneumatically driven tactile display (PTD) version of the TVSS, made up of 5 elements, a webcam, which acquires video images, connected to a software package (TVSSCs) implemented on a computer. The PTD control involves the PC with the dedicated software, a trigger and input/output transforming unit (TIOTU), and the electronic pneumatic interface (EPI) with 64 pneumatic switches.

64 pneumatic switches. The connection between TIOTU and EPI provides power and control signals for the pneumatic switches. The EPI converts the electronic signals to pneumatic pulses and distributes the air flow onto tubes which are connected to a regular square matrix $(80 \times 80 \mathrm{~mm})$ of $8 \times 8$ taxels (stamps of $1 \mathrm{~mm}$ in diameter covered by a plastic tip in contact with the skin and driven by a piston-see figure 2) placed on the subjects' abdomen. Each one of the taxels has a maximum temporal resolution of $50 \mathrm{~ms}$ and can be addressed individually with a maximum frequency of $20 \mathrm{~Hz}$. Taxels are either "on" or "off". A taxel is "on" when the stamp exceeds its shaft and "off" when it is inside (see figure 2). The air pressure from an external compressor can be adjusted by means of a pressure regulator, and can be varied between 3 and 7 bars, according to the subjects' tactile sensitivity.

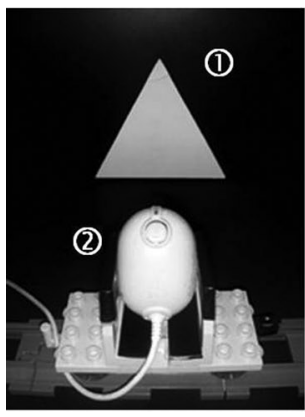

(a)

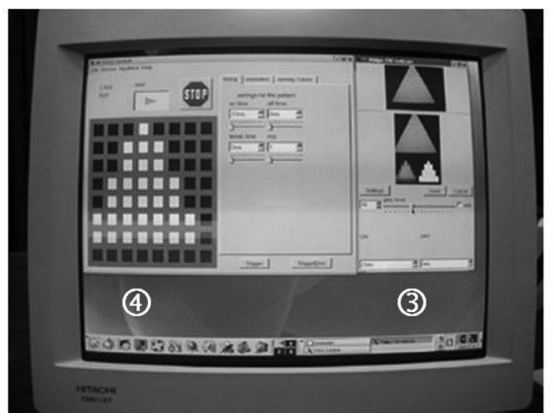

(b)

- Triangular cue displayed against a black screen 2 USB webcam of the PTD

3 Control screen for the webcam image and corresponding degraded image in $8 \times 8$ pixels

(4) Appearance of the tactile stimulation as seen on the experimenter's monitor

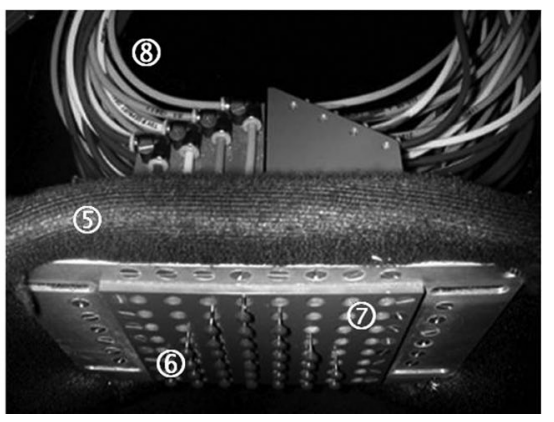

(c)

5 Belt to mount the matrix on the abdomen

6 Taxels in position "on"

$(7$ Taxels in position "off"

8 Plastic tubes for air flow

Figure 2. Example of the correspondence between a visual stimulus (a) and the tactile image [degraded image in $8 \times 8$ pixels (b), and activated taxels of the matrix (c)]. The EPI converts the electronic signals to pneumatic pulses and distributes the air flow onto tubes which are connected to a regular square matrix $(80 \mathrm{~mm} \times 80 \mathrm{~mm})$ of $8 \times 8$ taxels. 
Tactile perception, when using the PTD, is constrained by the subject's movements with the camera. Indeed, nervous excitation appears only if pressure modifications occur; perception is associated with the renewal of mechanical deformation induced by moving. However, the "on/off" position of the taxels depends on changing levels of the recorded image according to these movements.

\subsection{Maze and robot}

The maze and the robot were the same as the ones used in our previous study (see Segond et al 2005). Both, the mobile robot ("mobile of sensory-motor coupling", MSMC) and the TV camera mounted on it are remotely controlled by high-frequency connections. The camera is a $120^{\circ}$-angle lens mounted at the front of the robot. It collects the visual information in the maze and transmits it to the TVSS in order to display it on the PTD matrix. Subjects used two joysticks. The first enabled them to control vertical scan of the camera in order to perform fuller discrimination of visual stimuli and cues. The second enabled them to control movements of the MSMC by three means: forward movements, backward movements and rotations in either direction by the front wheel turning at an angle of $160^{\circ}$.

The maze's zones, separated by "saloon"-type double doors, were different in terms of difficulty level owing to their spatial configuration. Thus, the areas 2, 3, and 4 presented similar configuration but constituted a more complex configuration as regards the starting room (first area) - they were smaller than the starting room. The number of areas to explore was reduced from 5 to 4 as in our study with blindfolded sighted subjects using the ETV4 version of TVSS (Segond et al 2005). Despite a higher density of tactors for the ETV4 compared with the PTD versions, we noticed an important alteration of performances in the 5th area due to the highest complexity level of this area (cf little room inserted into a larger one). We wished to avoid such degree of complexity for blind and blindfolded sighted subjects using a matrix with only $8 \times 8$ tactors in the present study.

\subsection{Procedure}

The TVSS training was made up of 5 distinct trial blocks (see table 2). Subjects underwent 20 sessions of training, with an average duration of $1.5 \mathrm{~h}$. Throughout the study, the subjects were given the opportunity to experience spatial phenomena (normally perceived by visual stimulation), via the vibrotactile stimulation. This topic was addressed by three main phases: first, we explored the possibilities for subjects to locate and identify distant 2-D shapes with the 64-taxel device (trial blocks 1, 2, and 3); second, we aimed to find out whether the device could permit the experience of spatial phenomena-especially monocular depth cuesand whether subjects could learn to recognise shapes from partial views (trial block 4); finally, we assessed if the subjects could apply these newly acquired vibrotactile pieces of information to a truly 3-D environment in a maze navigation task (trial block 5). Subjects worked seated at a table and the PTD matrix was worn on a belt around the waist, in contact with the skin on the abdomen. According to the aim of each session, the camera was placed in the following fashions: (i) on a mobile base manipulated by the subjects along a straight rail, which allowed the camera to perform a vertical scan associated with a lateral movement from left to right and vice versa (rail I: placed horizontally in a fronto-parallel plane- see figure 3), or a forward-backward movement (rail II: placed perpendicularly to the frontoparallel plane - see figure 4a); (ii) on a mobile base manipulated along a semicircular curved rail (rail III: only the scan through the rail was available - see figure 4b); (iii) on a remotecontrolled robot (vertical scan of the camera and movement of the robot were permitted by a high-frequency connection between the robot and the TVSS - see figure 5).

Before starting every session, the sighted subjects were blindfolded. They never saw the experimental setting. They could only explore it haptically, just like the blind subjects. 
Table 2. Training procedure.

Trial block 1: familiarisation and development of perceptual motor loops

Trial block 2: detection and recognition of lines and line combinations

Trial block 3: identification and recognition of 2-D shapes

Trial block 4: shape recognition from perspective views and depth movement

Trial block 5: application to depth and space perception; maze navigation task session1: horizontal and vertical scanning-familiarisation to the reversal moving of the object

session 2: detection and identification from object's characteristics

session 3: pre-test

session 4: recognition of lines and line combinations with 2 elements session 5: recognition of line combinations from 2 to 3 elements session 6: recognition of line combinations from 3 to 4 elements session 7: post-test

session 8: recognition of simple geometrical shapes session 9: recognition of simple geometrical shapes session 10: recognition of simple geometrical shapes session 11: recognition of simple geometrical shapes

session 12: recognition from perspective views session 13: recognition from "zooming" effect session 14: recognition from perspective views session 15: recognition from "zooming" effect session 16: familiarisation with task session 17: maze cues recognition session 18: simplified maze navigation task session 19: test 1 (first maze navigation) session 20: test 2 (second maze navigation)

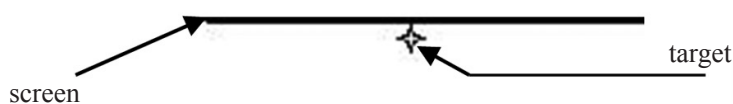

(1)

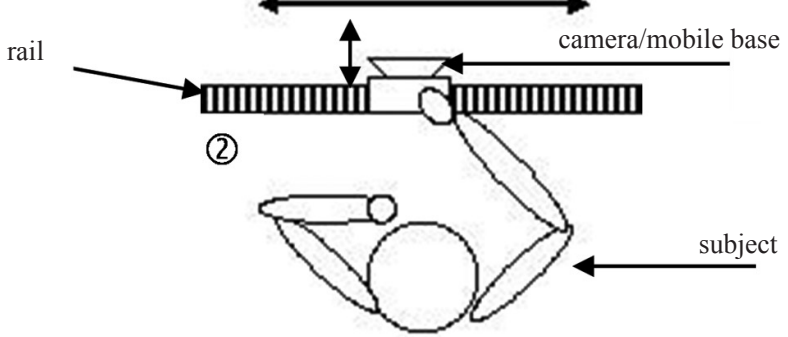

Figure 3. Aerial view of the experimental display with a straight rail allowing the camera to perform a vertical scan (2) associated with a lateral movement (1) from left to right and vice versa (rail I: placed horizontally in a fronto-parallel plane).
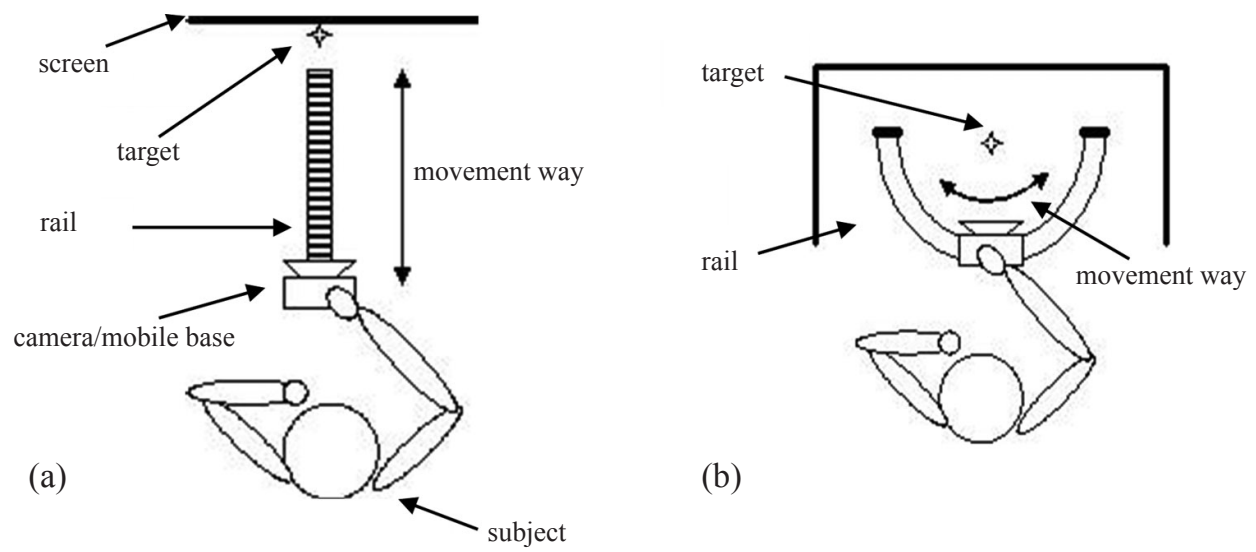

(b)

Figure 4. Mobile manipulated by the subjects along (a) a straight rail allowing the camera to perform a forward-backward movement (rail II: placed perpendicularly to the fronto-parallel plane); (b) a semicircular curved rail (rail III: only the scan through the rail was available). 


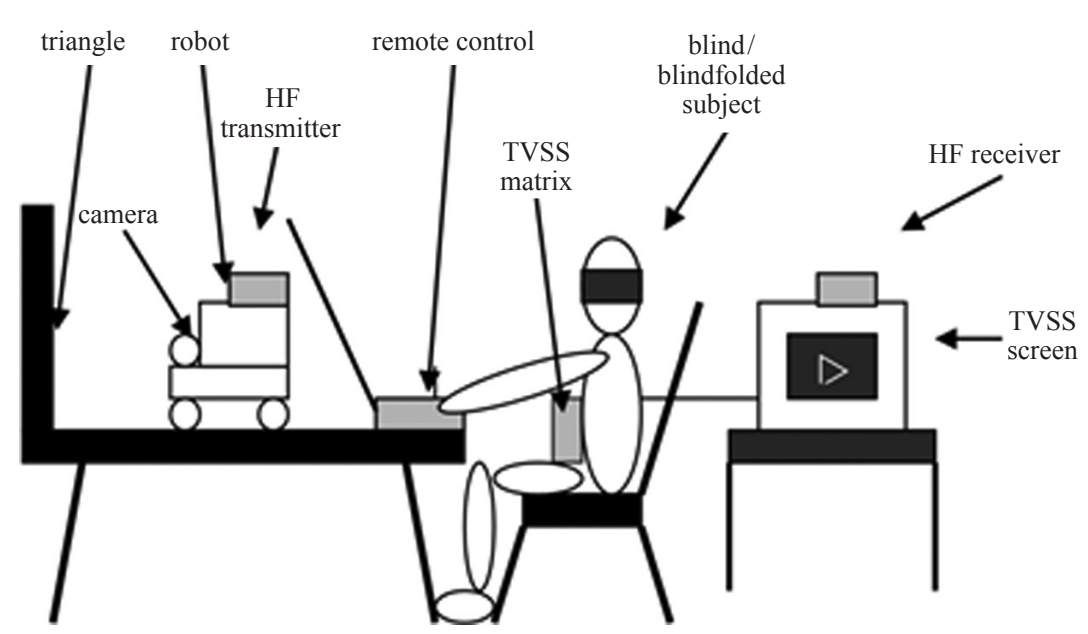

Figure 5. TVSS camera placed on a remote-controlled robot [vertical scan of the camera and movement of the robot were permitted by a high-frequency (HF) connection between the robot and the TVSS].

Throughout the learning, white stimuli were displayed against a black background to enhance pattern-ground contrast. In order to optimise the development of new visuo-tactile abilities, verbal and haptic feedback was provided subsequently to each learning sessions trial (except in test sessions). When the subjects failed to identify the presented stimuli, they could explore them haptically (eg magnetised sticks, wooden forms), and then, once again, through camera.

2.4.1 Learning about localisation/identification of single or combined lines varying in orientation and 2-D geometrical figures. Training trial blocks 1 (sessions 1 and 2), 2 (sessions 3 to 7), and 3 (sessions 8 to 11). The first and second sessions (trial block 1) were dedicated to familiarisation with the experimental surroundings, tactile stimulation, and the camera scan. The principle of the device was explained to the subjects (ie the transmission, in real-time, of the filmed image onto the skin). This part of the first learning trial block focused on developing accurate and reliable perceptual-motor control and providing sensory motor skills to optimise the camera scan. Subjects were able to scan the visual field in two directions (up/down, left/right) by moving the camera along rail I (see figure 3 ). The distance of the rail/camera from the pattern was kept constant $(45 \mathrm{~cm})$. In the first stage, they learnt to use horizontal scan only, then vertical scan only, and then the two coupled to each other. Subjects were also told of the reversal phenomenon related to the camera movement (when the camera was moved from left to right, the image seemed to move from right to left).

They were given simple tasks to familiarise themselves with this reversal phenomenon. For instance, in session 1 they were shown the direction of movement of a programmed shape, and then told to reproduce its movement with an appropriate hand gesture and camera movement. The role of trial block 1 was very important. Subjects could freely explore the visual field and therefore detect sensory-motor correspondences, to test and develop their own scan strategies. This camera handling was essential because subjects had to keep moving it, in order to perceive the tactile stimulation relative to the position "on" or "off" of the taxels. Later on, subjects moved on to the detection and recognition of 2-D patterns (trial blocks 2 and 3).

Subjects began with trial block 2 (sessions 3 to 7). The stimuli used were 28 patterns of single lines (14 cm long and $2 \mathrm{~cm}$ thick) or combined lines varying in orientation and complexity (see figure 6). Subjects had to detect patterns and identify them, one at a time, with a maximum of 3-min exploration time. 


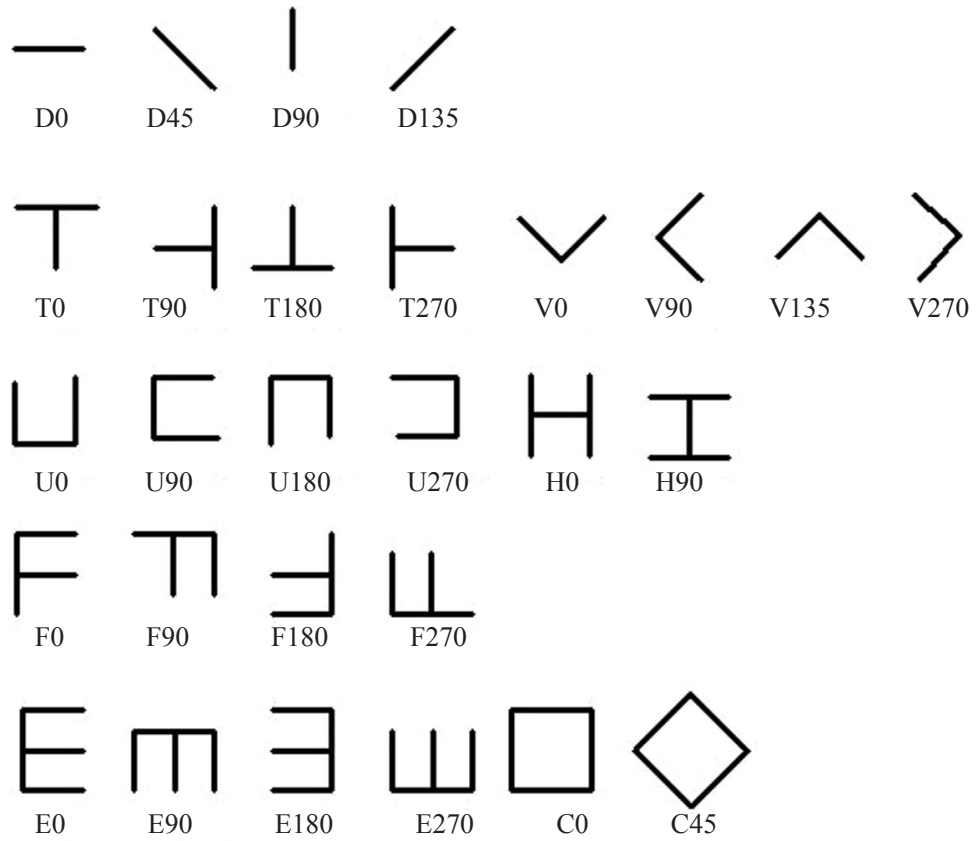

Figure 6. The 28 used patterns. Each of them is designed by a code (letter and rotation angle).

The training trial block 2 consisted of 5 consecutive sessions: 3 training/learning sessions presented between 2 test sessions T1 (pre-test) and T2 (post-test). The detailed scheme of training sessions is presented on figure 7. Each training session consisted of 8 trials ( 8 patterns per session). 4 of the 28 patterns were exclusively used in the test sessions in order to determine the subjects' identification abilities with new patterns.

Training began with the easiest stimuli [1 or 2 line(s) at the first session] and followed through the most complicated ( 2 and 3 lines at the second session, 3 and 4 lines at the third session). A test was presented at the end of each training session, where subjects had to

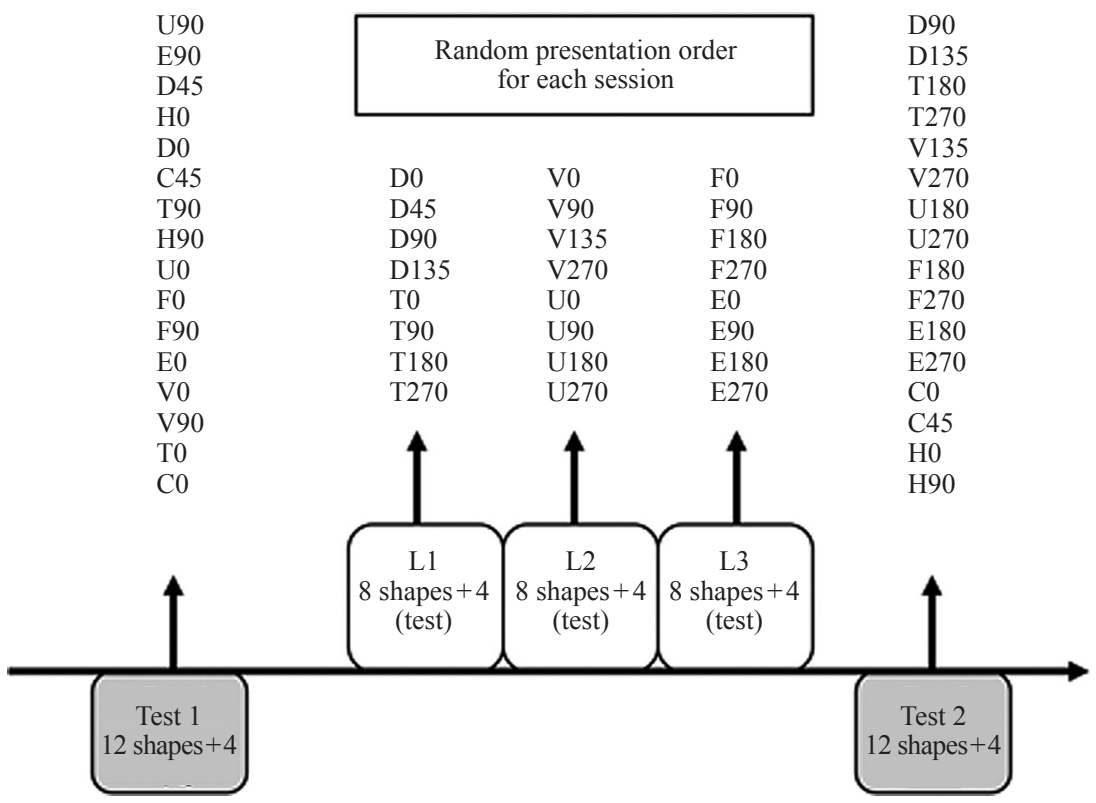

Figure 7. Design of the study for the 5 sessions of trial block 2: 3 training/learning sessions (L1 to L3) and 2 test sessions ( $\mathrm{T} 1$ and $\mathrm{T} 2$ ). 
identify 4 items already discriminated during the training. The aim of this test was not only to reinforce the learning but also the subjects' motivation by providing an easier task. Subjects were asked to give a concrete form to their answers by constructing the pattern they had explored using magnetised sticks on a magnetised tablet (procedure inspired by the one used by Arno et al 1999, 2001). Training was reinforced by tactile feedback after each trial: when the subject's answer didn't correspond at all or only partially to the "model", the good configuration was presented to the subject on the tablet. Thus, the subject could haptically explore the modified configuration on the tablet and then via the camera again.

Trial block 3 was designed to learn identification of triangular geometric forms in order to prepare subjects for recognising the triangular cues found in the maze of the final navigation tasks (trial block 5). In each learning session of this block (from session 8 to 11), subjects had to determine the orientation of an isosceles triangle (ie to designate which direction the vertex was pointing to-see figure 8 ), with a maximum of 3 min exploration time.

The same isosceles triangle was presented in one of the four following orientations: up, down, right, and left. These four cues (raised wooden forms) were first presented to the subjects with a view to being explored haptically. The first 3 sessions ( 8 to 10) were carried out in the same conditions as the previous ones (on rail I; cf figure 3). As for the last session of the trial block 3 (session 11), the camera was placed in front of the remote-controlled robot (see figure 5) as it was during the navigation tasks. Only vertical scan was allowed and made via a remote control.
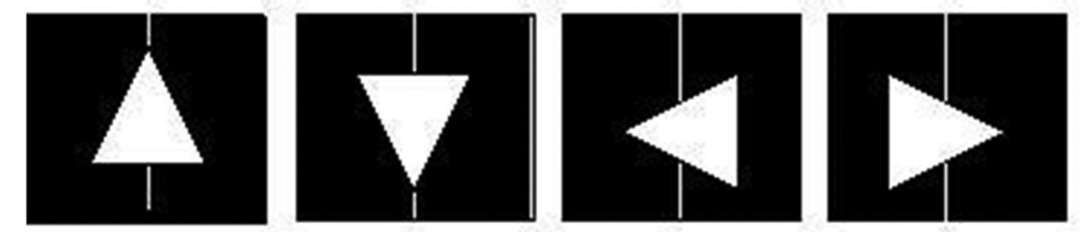

\begin{tabular}{|c|c|c|c|}
\hline Vertex upward: & Vertex downward: & Vertex to the left: & Vertex to the right: \\
\hline to move ahead & to move backward & to move leftward & to move rightward \\
\hline
\end{tabular}

Figure 8. 2-D triangular targets presented during training and associated with directional cues on the maze doors, indicating the way to follow.

\subsubsection{Learning about identification of distant 2-D shapes on the basis of monocular depth cues:} training trial block 4 (sessions 12 to 15). The majority of the training dealt with depth perception and the interpretation of 3-D space by the use of monocular cues via the visuotactile substitution system. Differences and similarities between vision and touch/haptic concerning the relation between 2-D stimulation and 3-D perception are well documented in the literature, particularly through the contribution of authors such as Berkeley, Helmholtz, Gibson, and Jansson (see, for review, Delorme 2003).

According to Collins and Bach-y-Rita (1970), the cues most readily available for thirddimension judgments with the TVSS include linear perspective, apparent height of object base in the visual field, size change, clarity, and interposition.

Consequently, in the trial block 4 (session 12 to 15 ), subjects could only experience successive views of the triangle (ie linear perspective) and the outside contour modification of the shape with the distance variation (ie apparent size change) in order to identify the geometrical form (the same triangle in four basic positions as above). These phenomena were investigated in two generalisation tasks for object recognition: (i) a perspective effect task where the camera was allowed to perform rotational movements around the shape thanks to the semicircular curved rail (rail III; cf figure $4 \mathrm{~b}$ ); this generated a constantly changing 
perspective view of the triangle (from isosceles triangle view when the camera is in front, to a vertical-line view when the camera is positioned perpendicularly); (ii) a "zooming"effect task where the camera could perform forward-reverse movements on the straight rail positioned toward the object (rail II; cf figure 4a). This movement led to variation in the outer contours of the presented triangle. The relative size of the pattern increased when the subject moved the camera closer to the stimulus and decreased when he/she drove the camera away. In the course of the 2 tasks ( 2 sessions per task according to a counterbalanced order between subjects), subjects had to identify the patterns, one at a time (maximum: 3 min exploration time), giving their response verbally.

\subsubsection{Using optical cues and depth perception capacities in a navigation task: training trial} block 5 (sessions 16 to 20). The main objective of this trial block was to determine if, and to which extent, subjects could apply their newly acquired knowledge and strategies concerning forms and depth by visuo-tactile perception to a 3-D environment. We examined the subjects' spatial orientation by means of a maze navigation task where a radio-controlled robot was driven with joysticks. This kind of task brings a dual advantage: it allows evaluating the subjects' possibilities to "travel in space" and put into work acquired recognition abilities in a safe orientation task offering also an aspect of a play.

The progress of learning acquisition was gradually evaluated in 5 stages (from sessions 16 to 20). The first 2 sessions of trial block 5 (16 and 17) aimed at familiarising the subjects with the handling of the robot and the camera controls (described in section 2.3), then with the perception of the cues in the maze. 3 types of cues were placed to help the subjects get bearings in the maze. (1) The on-the-door 2-D triangular cues indicated which direction and possibly which door had to be taken. Triangles, initially explored with different camera movements, were then used as direction cues. Thanks to the orientation of the triangle, the subjects were informed whether they could cross the doors (upwards orientation), or, if not, they were informed about the direction they had to follow (to the right, to the left, or to turn round facing downwards orientation). (2) Lateral lines on the room walls indicating that the robot was in front of an obstacle. (3) A continuous line on the floor along the corridors was used to go through the corridors from room to room. Lateral line discontinuity (ie empty space between the end of a line — end of the wall — and the closer on-the-door triangle) could be regarded as the 4th cue. Practising exercises were previously presented to the subjects (session 16). They were to command movements to the robot placed on the table and within the reach of the subject's hands, so that they felt the different moving speeds and followed its trajectory. In the next task (session 17), the robot was placed in the large starting room of the maze: for some trials, it was opposite the triangle (position 1); for others, it was on a slant to the right or left according to position 1 (position $2:+45^{\circ}$ angle, position $3:-45^{\circ}$ angle) causing a perspective view of the cue (see figure 9).

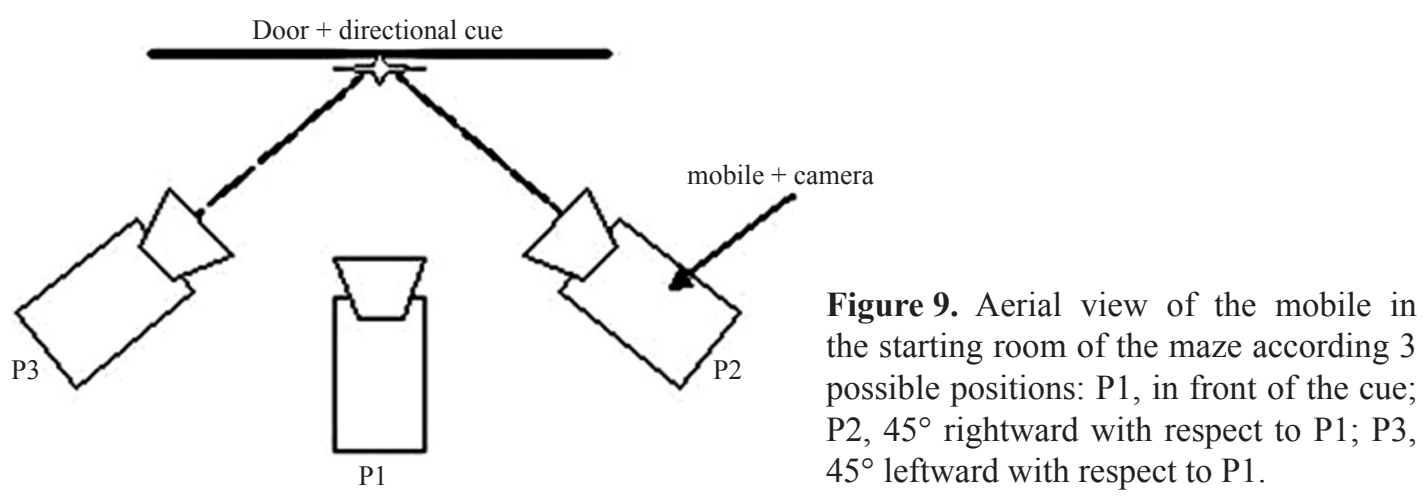


The subjects had to identify the orientation of the triangle by using 1 of the 3 kinds of explorations made available: E1 - vertical scan of the camera while the robot was stationary, E2 - forward-backward movement with no vertical scan (ie "zooming effect"), E3 - both movements (vertical scan+zoom). Prior to each trial, the subject knew the position of the robot and the scan movement he/she could perform. A simplified navigation task in the large maze room was used to introduce the subjects to the maze navigation task, to facilitate it as much as possible (session 18). Finally, 2 successive maze navigation tasks were used according to 2 different routes with the same complexity level (sessions 19 and 20). Each route was composed of 4 exploration areas. These subdivisions offered us the possibility of analysing the evolution of performance between the areas in a single navigation and between the 2 navigations (see Segond et al 2005).

Subjects were asked to go from the large starting room to the exit of the 3rd of 3 small rooms, by using the cues. The subjects had to make a series of choices and answer several questions in order to find the right path. (1) Detection: Is there something in front of me? Where is it (within the camera field)? (2) Identification: What is its size? How far is it? What cue is it? (3) Orientation: In what direction do I have to go? Which movements do I have to perform? To follow the progress of the task with respect to the subjects' point of view, they were asked to relate as much as possible what they perceived and which actions they decided to take (their speech was recorded). It allowed us to determine the processing time for each cue. In order to neutralise the size disparity and thus to be able to show a training effect by comparing exploration times, the sizes of all zones in the maze were set to a single $1 \mathrm{~m}^{2}$ surface unit for analyses.

\section{Results}

The findings showed generally that early blind and blindfolded sighted subjects were able to gradually carry out the tasks of a perceptual learning programme with a 64-taxel pneumatic system (PTD). They exhibited significantly improved performance and finally succeeded in the navigation tasks.

The subjects' perceptual motor skills improved greatly, qualitatively, in the course of navigation tasks (eg from the subjects' verbalisation). Subjects acquired coordination skills to control the robot/camera couple according to the information perceived. Most subjects reported that the second navigation task seemed easier and proved themselves more proficient in the handling of the robot. The task was performed with more ease; the speed of the robot movements was well mastered. Therefore, too fast movements were prevented, whereas localisation and exploration of triangular cues were enhanced. During the room exploration, strategies of several blind and sighted subjects appeared systematic. Using alternately backwards then forwards movements while turning to the right or to the left, subjects attempted to keep a central position of the robot in the room. Thus, they maintained the robot at a suitable distance from the walls and the doors of the maze. Most of them had the robot moving along the horizontal line using it as a guideline, thereby detecting the following door. Two sighted subjects also used a strategy developed during learning, such as the exploration of the triangle's isolated right/left side, which was explored with vertical movements. Most subjects better understood the logic in the cue layouts in the course of the second navigation (ie empty space after a lateral line followed by a triangle). Indeed, this piece of information was taken into account by only four of the subjects in the first navigation, all being sighted. In the second navigation, eleven subjects - five blind and six sighted - could rely on these spatial-cues relationships to perform the task more swiftly. This understanding of cue organisation permitted subjects to have a representation of the task in terms of route. Moreover, they expressed themselves - particularly in the course of the 
second navigation - as if they were actually in the maze and were carrying out maneuvers physically. All the subjects - except one blind - spontaneously used the word "I" to describe what they experienced: "I am already in front of a triangle", "I can leave the room", "I'm going towards the right side", "I'm stuck!", "Ah, ah... I'm closer"; "I must turn back!"

After this general qualitative presentation of the results, it is now time to show more detailed analyses of training procedure performances of the subjects. Most of the following statistical analyses were conducted separately on response accuracy, after transformation of the rate into arc-sinus, and processing time.

3.1 Familiarisation to the experimental setup and to perceptual motor control (trial block 1) The two initial tasks were performed easily. Subjects could experience the changes in stimulation relative to the vertical scan and the camera movement along the rail I. They could rapidly perceive when a target was partially filmed and had no difficulty in centring the image on the matrix by manipulating the camera. Once familiarised with the handling of the camera and the necessity of taking into consideration the reversal phenomenon of the image with the camera moving, the subjects rapidly verbally described the movement of a programmed target and could easily imitate it. The sighted subjects could refer to a similar phenomenon, which occurs in vision. Likewise, blind subjects encountered no difficulties when becoming familiar with the reversal phenomenon. The blind subjects who best adapted to this phenomenon referred to similar experience in their everyday life (eg the use of the computer page-up and page-down keys). Mean and standard deviations of correct responses and processing time in the next trial blocks $(2,3,4$, and 5) are presented in table 3.

Table 3. Mean (standard deviations) percentage of correct responses and processing time for a group of subjects (13 subjects), blind subjects, and blindfolded sighted controls in the main tasks of trial blocks 2, 3, 4, and 5 .

\begin{tabular}{|c|c|c|c|c|c|c|}
\hline & \multicolumn{3}{|c|}{ Percentage of correct response } & \multicolumn{3}{|c|}{ Processing time $/ \mathrm{s}$} \\
\hline & $\begin{array}{l}\text { all } \\
\text { subjects }\end{array}$ & $\begin{array}{l}\text { blind } \\
\text { subjects }\end{array}$ & $\begin{array}{l}\text { sighted } \\
\text { control }\end{array}$ & $\begin{array}{l}\text { all } \\
\text { subjects }\end{array}$ & $\begin{array}{l}\text { blind } \\
\text { subjects }\end{array}$ & $\begin{array}{l}\text { sighted } \\
\text { control }\end{array}$ \\
\hline \multicolumn{7}{|c|}{ Trial block 2 (line combinations) } \\
\hline test 1 & $46.1(7.5)$ & $45(11.1)$ & $47.6(4.5)$ & $140(22)$ & $144(27)$ & $137(34)$ \\
\hline test 2 & $56.4(8.5)$ & $53.2(7.5)$ & $59.1(9)$ & $148(25)$ & $147(29)$ & $149(30)$ \\
\hline \multicolumn{7}{|l|}{ Trial block 3 (triangles) } \\
\hline means (sessions 8 to 10 ) & $63.5(22.2)$ & $52.4(22)$ & $72.7(22)$ & $105(39)$ & $123(31)$ & $89(36)$ \\
\hline session 11 (robot) & $80.7(7)$ & $81.8(4.6)$ & $79.7(8.9)$ & $62(25)$ & $80(32)$ & $45(13)$ \\
\hline \multicolumn{7}{|l|}{ Trial block 4 (depth cues) } \\
\hline perspective effect & $52.4(14.8)$ & $41.2(6.2)$ & $62(18.9)$ & $94(22)$ & $97(26)$ & $92(29)$ \\
\hline zooming effect & $75(14.8)$ & $66.2(9.2)$ & $82.4(13.2)$ & $70(28)$ & $81(21)$ & $61(23)$ \\
\hline \multicolumn{7}{|l|}{ Trial block 5 (maze) } \\
\hline first navigation & $72.8(15)$ & $68.9(16)$ & $76.6(14)$ & $127(40)$ & $129(40)$ & $125(40)$ \\
\hline second navigation & $78(13)$ & $78(12.5)$ & $78(14)$ & $105(45)$ & $120(53)$ & $92(40)$ \\
\hline
\end{tabular}

\subsection{Identification of lines and of line combinations (trial block 2)}

The subjects' performance was analysed in a 2 (group: EB versus SC) $\times 2$ (test session: T1 versus T2) ANOVA. The normality of distributions thereby obtained was checked empirically and confirmed by means of Kolmogorov-Smirnov statistical tests. The degree of success was evaluated as a percentage in regards to the degree of similarity between the subjects' construction and the "model" (shape, size, and orientation). As in the study of Arno et al (1999), 
we used a tracing quotation by superimposing the two patterns (as several overlays were possible, the best correspondence was retained for each trial, without applying any rotation). We determined the number of common points (pixels) between the subject's response and the presented pattern by considering a magnetised stick as 7 adjacent collinear pixels/ taxels, that is to say 7 consecutive taxels in "on" position. The number, $X$, of common points (pixels) between the stimulus pattern, $S$, and the subject's response, $R$, was determined and the score (rated from 0 to 1 ) was given by the ratio $X /(S+R-X)$. The analysis revealed the significant main effect of the test session factor $\left(F_{1,11}=6.70, p<0.05\right)$ but no effect of the group factor. Thus, response accuracy of both groups significantly increased between $\mathrm{T} 1$ and T2. The analysis on processing time exhibited no main effect of group or test session factors. However, the analysis of individual subjects' responses revealed that inter-individual variability exists in the ability to perform the task in both groups. Some subjects (two blind and one sighted) showed higher response accuracy from the first test session, while other subjects scored approximately half as well as the most efficient subjects. These differences regarding both groups' performance were still visible in T2, although to a lesser extent. Qualitative analysis allowed us to bring to the fore differences in subjects' strategies during pattern exploration.

Four key strategies could be distinguished among the latter ones. First strategy: exploration of well-centred pattern with performing distinct horizontal and vertical movements. Second: "scanner-like" exploration of the pattern with associated horizontal and vertical movements. Third: exploration of patterns' sides, one by one, isolated right/ left side by vertical movements and bottom/top side by horizontal movements. Fourth: exploration of the global form by performing slight horizontal movements to the left and to the right as in a vibratory mode.

During T1 and T2, the strategies appeared very systematic. In T1, all subjects explored the well-centred/framed pattern with alternating horizontal and vertical movements (strategy 1). In the course of the three learning sessions, most of the subjects spontaneously developed and used some of the other strategies. Finally, in T2, the patterns were explored by all subjects by using the first and/or the third strategy (eight subjects used both of them). The first strategy seemed to be used to perceive if the pattern contained a horizontal, vertical, or oblique line. The third strategy presumably helped them to analyse how the lines were spatially organised.

\subsection{Identification of triangular shapes (trial block 3)}

A Pearson correlation coefficient computed between the performance and processing time of the two groups (blindfolded sighted and blind subjects) revealed that an inverse relationship existed $(r=-0.70)$. Consequently, the most efficient subjects in this task needed less time to recognise the different triangles.

Performance was analysed in a 2 (group) $\times 3$ (learning session-from session 8 to 10 ) ANOVA. This analysis suggested no main effect of group or of test session factors, so the learning effect was not obvious. This may be explained by observed individual differences of performance. The analysis of mean individual performance allowed bringing out two distinct categories of subjects. A "high" category comprises subjects who scored at least $70 \%$ correctly (five sighted and two blind subjects). A "medium" category comprises subjects who scored less than $50 \%$ of correct replies (the other two sighted and four blind subjects).

The results stemming from session 11 were analysed separately, because the handling of the camera was different. The transition from rail scan to scan via joystick was well managed by all the subjects. The performance of both groups did not differ concerning mean percentage of correct responses, but was significantly different in processing time. The sighted subjects were faster to provide their responses in this session than the blind subjects $\left(t_{11}=2.25\right.$, $p<0.05$ ), except for the two blind subjects who were part of the "high" group. 


\subsection{Perspective and zooming effect tasks (trial block 4)}

Performance was analysed in a 2 (group) $\times 2$ (session) ANOVA for each task.

The ANOVA on response accuracy concerning the zooming effect task revealed the significant main effect of the group factor $\left(F_{1,11}=5.09, p<0.05\right)$. There was no effect of the session factor. Further analysis revealed that performance was significantly better for sighted subjects than for blind subjects in the first session (ie session 13) of the zooming effect task $\left(t_{11}=-2.73, p<0.05\right)$. This difference was no longer visible in the second session of the task (ie session 15; see figure 10). Performance of the sighted group remained the same while performance of the blind group significantly increased from the first session to the second $\left(t_{11}=-3.05, p<0.05\right)$.

The ANOVA on response accuracy concerning the perspective task revealed the significant main effect of group factor $\left(F_{1,11}=6.24, p<0.05\right)$. There was no effect of the session factor. Further analysis indicated that performance was significantly better for sighted subjects than for the blind subjects in both, the first session (ie session $12 ; t_{11}=-2.24, p<0.05$ ) and the second session (ie session 14; $t_{11}=-2.3, p<0.05$ ). Performance of the sighted group remained the same, and performance of blind subjects did not increase in this task unlike in the zooming effect task (see figure 10).

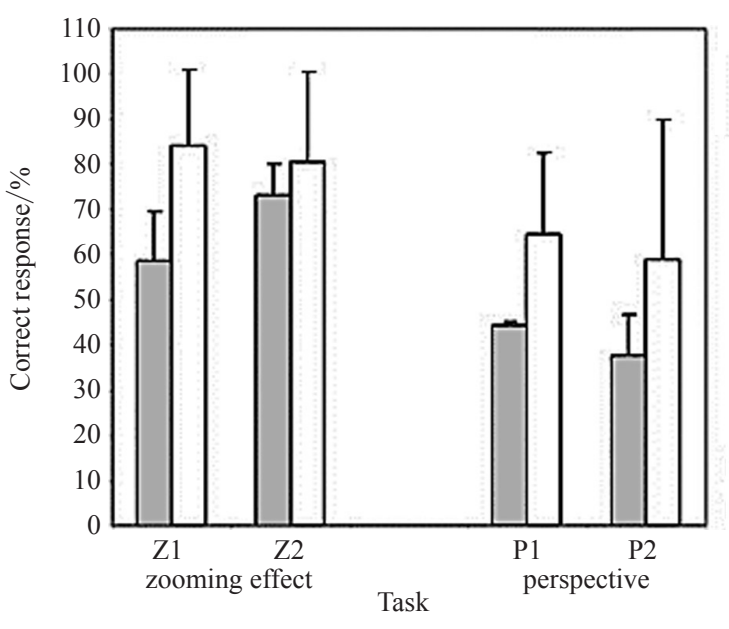

Subjects

$\square$ blind

$\square$ blindfolded sighted

\subsection{Maze navigation task (trial block 5)}

Prior to the analysis of response accuracy (ie correct identification of triangular cues) and processing time to carry out the two successive maze navigation tasks, we looked into the subjects' performance in the task of triangular cue recognition (session 17) from different available explorations (from E1 to E3; see section 2.4.3 and below) and robot positions (from P1 to P3; see figure 9). A 2 (group) $\times 3$ (exploration mode) $\times 3$ (position) MANOVA revealed the main effect of exploration mode factor $\left(F_{2,22}=8.89, p<0.05\right)$. There was no significant effect of the group or of the position factors. A further analysis indicated a significant difference between the vertical scan (E1) and the zooming mode (E2) $-p<0.05-$ as well as between the zooming mode (E2) and the vertical scan + zooming mode (E3) $-p<0.001$. Moreover, the analysis revealed the interaction of the group and exploration-mode factors $\left(F_{2,22}=4.89\right.$, $p<0.05)$. This reliable interaction indicated that performance was distinct according to the exploration mode. Blind subjects showed better performance when using only the vertical scan (E1) compared with the two other scan modes (respectively $\chi^{2}=5, p<0.05$ with the zooming mode E2, and $\chi^{2}=5, p<0.05$ with vertical scan + zooming mode E3). Sighted subjects, on the other hand, scored better performance when using vertical scan coupled with forwardsbackwards movements of the robot (E3) compared with the vertical scan $\left(\mathrm{E} 1 ; \chi^{2}=6, p<0.05\right)$ and forwards-backwards movements (E2; $\chi^{2}=5, p<0.05$ ) separately (see figure 11 ). 


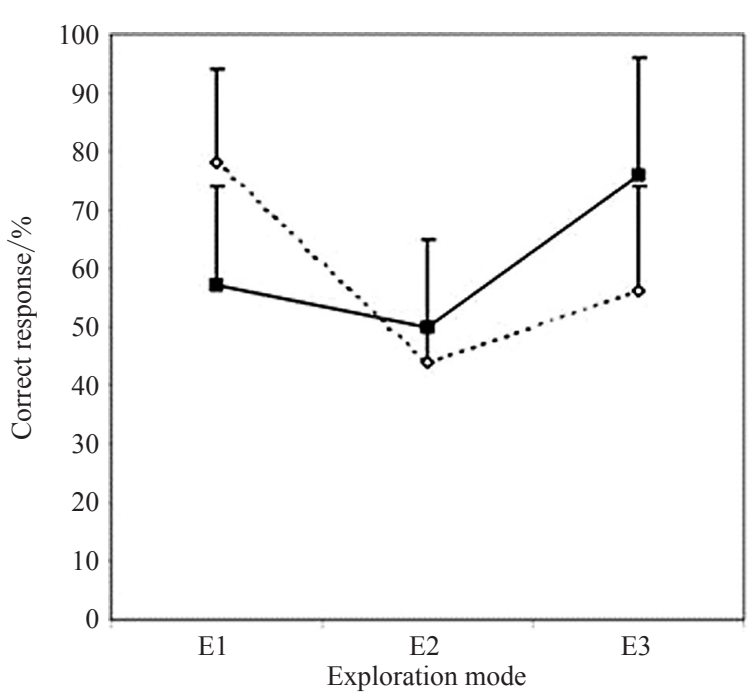

Subjects

...... blind

$\longrightarrow$-blindfolded sighted

Figure 11. Mean accuracy as a function of group and exploration mode. $\mathrm{E} 1=$ vertical scan of the camera only, E2 = forwardbackward movement only (ie 'zooming effect'), E3 = both movements (vertical scan + forward-backward movement). The SDs are represented by the error bars.

We can associate these results with the poorer ones obtained by blind people when compared with the sighted group in the perspective and zooming effects tasks. Performance of the blind group significantly increased from the first session to the second for the zooming task (sessions 13 and 15). However, the training procedure was probably insufficient for blind subjects to allow them to use spontaneously this exploration mode (E2; forwardsbackwards movements, relative to optic flow). This was testified by their lack of improvement in perspective task. This is probably due to the zooming process still being far from their everyday life experience in which they mostly use scan movements (cf white cane). So, in short, blind people struggled more because they had fewer concepts of the shapes they were "seeing".

All subjects succeeded in the following maze navigation tasks. On average, the blind subjects completed the first and the second navigations (sessions 19 and 20) in $63 \pm 14 \mathrm{~min}$ and $47 \pm 18 \mathrm{~min}$, respectively. The blindfolded sighted subjects completed the first navigation (N1) in $61 \pm 13$ min and the second one (N2) in $40 \pm 13$ min. A 2 (group) $\times 2$ (navigation) ANOVA revealed the significant main effect of the navigation factor. The mean time to succeed in the task was significantly shorter in the second navigation than in the first $\left(F_{1,11}=16.41\right.$, $p<0.05$ ).

In the following analyses, performance was analysed according to three recorded measures: (1) the accuracy of 2-D triangular cue identification (cf the rate of correct replies), (2) the average processing time per cue, and (3) the average time of the robot presence (exploration time) in each zone (exploration time was measured in time scale $\mathrm{m}^{-2}$ ). We conducted separately a 2 (group) $\times 2$ (navigation) $\times 4$ (zone) MANOVA on the three dependent measures.

3.5.1 The accuracy of triangular cue identification. The analysis of good replies showed that there was no influence of the different factors (ie group, navigation, zone) but suggested a significant interaction between the three factors $\left(F_{3,33}=3.28, p<0.05\right)$. Accuracy of cue identification by both groups was a function of zone and navigation (cf figure 12). Subjects showed good generalisation of learning of how to recognise, with accuracy, the triangular cues. The mean rate of correctly identified cues for the first session of navigation (session 19) was $68.9 \%(\sigma=16)$ for the group of blind subjects and $76.6 \%(\sigma=114)$ for the control group of blindfolded sighted subjects. For the second navigation session (session 20), this rate was $78 \%$ for both groups ( $\sigma=12.5$ and $\sigma=14$, respectively). Overall, these performances appeared much higher than the random threshold for both navigation sessions $\left(\chi^{2}=1356.72\right.$; $p<0.0001$ for $\mathrm{N} 1$ and $\chi^{2}=1574.48 ; p<0.0001$ for $\mathrm{N} 2$ ). 


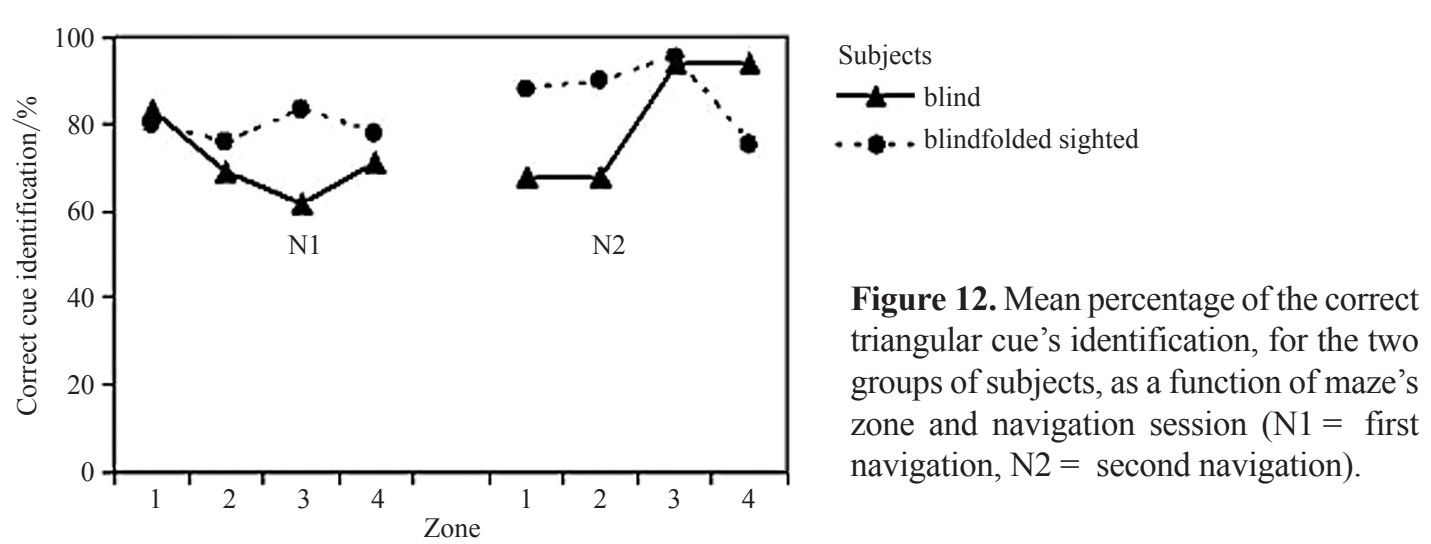

3.5.2 Average processing time per cue. There was a significant main effect of the zone factor on the processing time per cue $\left(F_{3,33}=8.74, p<0.001\right)$. As subjects advanced the robot zone by zone, the processing time of blind and sighted subjects in both navigations decreased. There was no effect of group or navigation factors. An a-posteriori analysis (Newman-Keuls test) showed that processing time during N1 significantly decreased between zones 1 and 2 $(p<0.05)$, zones 1 and $3(p<0.01)$, and zones 1 and $4(p<0.01)$. For N2, the decrease of processing time between zones 1 and 3 as well as zones 1 and 4 was significant $(p<0.05)$ (see figure 13).
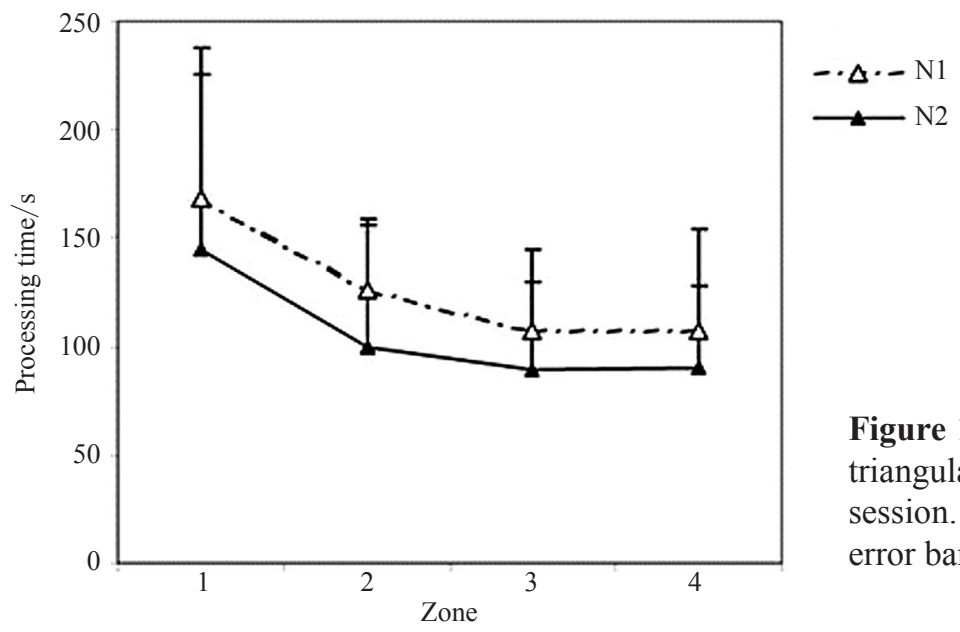

Figure 13. Mean processing time of the triangular cues as a function of navigation session. The SDs are represented by the error bars.

3.5.3 Average time of the robot's presence in each zone. The 2 (group) $\times 2$ (navigation) $\times 4$ (zone) MANOVA revealed a significant main effect of navigation $\left(F_{1,11}=17.89, p<0.05\right)$ and zone $\left(F_{3,33}=8.09, p<0.001\right)$ factors. There was no effect of the group factor. Further analysis showed that exploration time per zone significantly increased for N1 between zones 1 and $2(p<0.01)$, then significantly decreased between zones 2 and $4(p<0.01)$. However, it did not differ among the successive zones of N2. During N1, the subjects needed significantly more time to explore zone 2-which was smaller and of a more complex configurationthan they did in the large starting room and required an adaptation to this new space. The exploration time in N2 was steady among the zones (see figure 14). This observation gave evidence that the skill acquisition regarding space exploration was maintained when subjects performed N2. This result was confirmed by the analysis of the mean number of triangular cues explored in the course of both navigations. This number was significantly lower 


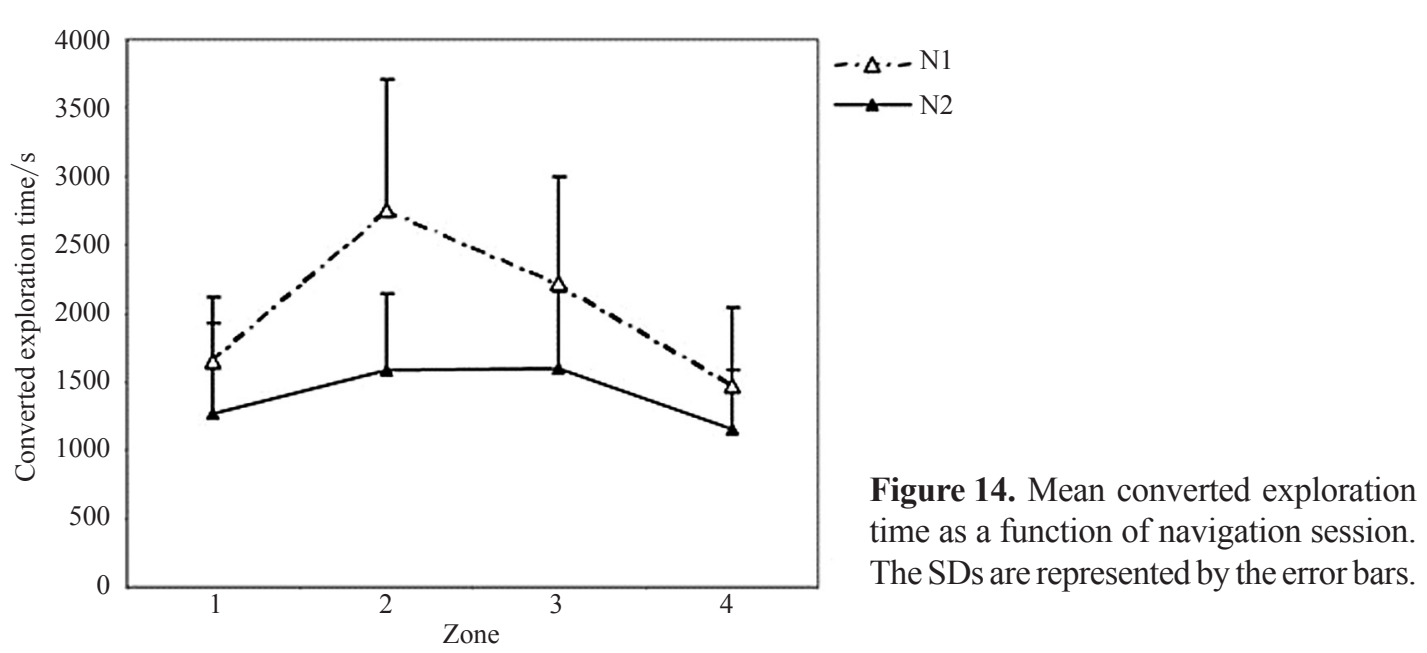

$\left(F_{1,11}=5.79, p<0.05\right)$ in $\mathrm{N} 2$ than in N1 (9.5 vs 12.1 on average for both groups), whatever the group.

\section{Discussion}

The results of the navigation tasks confirmed and extended our previous findings concerning sighted subjects, obtained with the 96-electrode ETV4 version of the TVSS in the same task (Segond et al 2005). Despite the smaller number of stimulation points, the subjects in this study were able to develop abilities in spatial localisation, shape recognition (with generalisation to different points of view), and monocular depth cue interpretation — especially useful during the navigation tasks.

During the training phase, early blind people initially experienced more difficulties than blindfolded sighted subjects (having previous perceptual experience of perspective) with interpreting and using monocular depth cues. In accordance with previous experiments on the perception of perspective in the blind (eg Hatwell 2003), this phenomenon suggests that blind subjects' poorer performance might be attributed to decreased understanding of this type of optical information than to the worse perception of tactile stimulation. Our results reflect those obtained in studies on blind and sighted people's mental imagery related to depth. For example, Arditi et al (1988) showed that only sighted people are able to imagine objects by adapting their size according to the distance between a subject and a represented object. Blind people imagine objects as placed in the near space, close to hand (Arditi et al 1988). However, and similarly to the results of Heller and collaborators $(1996,2002)$ showing the ability of congenitally blind subjects to learn the meaning of some aspects of perspective from foreshortened drawings, the amelioration of the performance for all blind subjects during our training sessions and the quite similar level of performance reached by two groups in the final navigation tasks suggested that early blind people were able to develop and apply cognitive understanding of depth cues. One of the blind persons reported that due to the training procedure and participating in our experiment, he eventually understood that optical information is not always visible. He referred to the fact that, as a child, he could not understand why sometimes his father was not able to read for him the advertising boards located at a certain distance in a street. Now he understood that distant perception depends on both, the position of the "observer" and the distance between him and the visual target.

During the navigation tasks, the participants of our study subjectively experienced shapes facing them. This externalisation appeared related to the way the subjects perceived themselves in relation to the explored space. Indeed, with training, people felt involved in the explored space (ie localisation of objects and events relatively to a point of view 
belonging to this space-Auvray et al 2005). Thus, both blindfolded sighted and early blind people mentally built a space by exploring the maze step-by-step and discriminating cues via the remote control robot. Perceiving shapes being located in the extra-corporeal space, using TVSS, refers to the pioneer work by Bach-y-Rita and his colleagues (Bach-y-Rita 1972; Guarniero 1974, 1977). They showed that this subjective experience appeared after training thanks to subjects' self-initiated actions on the camera, allowing establishing the stable correspondence between their actions and consequences of them. The recognition of perceived objects is based on the detection of perceptual motor invariants indicating a search by the action of the subjects and the mental construction of constant connections between action and feeling (Lenay et al 2000). In other words, this phenomenon underlines the importance of movement for space perception, as stated by Gibson $(1950,1979)$ for the visual modality. The dynamic change of images corresponding to the explored patterns, due to observer's or object's movement, delivers information on objects in the space and thus about the 3-D quality of the environment (Gibson 1950, 1979).

Our data show that subjects' performance depends not only on their perceptual experience, but also on their previous spatial competences. Indeed, we distinguished among early blind participants a two-person subgroup performing similarly to blindfolded sighted people. McLinden (1988) found alike that some blind people do not present the usual difficulties in spatial tasks. All our blind participants moved autonomously and had a job and/or social activities. However, the two constituting the "high" subgroup presented a superior degree of autonomy and played a team sport for blind people. The higher level of performance exhibited by two blind participants in our study could be, in correspondence with Hatwell's hypothesis (2003), associated with the bigger autonomy, ease of spatial mobility in their environment, and consecutive development of good spatial representation abilities.

Finally, the navigation tasks revealed that subjects manifested a particular attraction to the upwardly opened triangular cues. When recognising them, their comments demonstrated an undeniable jubilation. We wonder whether, as a previous experiment indicated (Segond et al 2005), subjects acceded to the qualia (ie emotional content of tactile stimulation) of sensory experience in the course of this task (see Bach-y-Rita 1995, 1997; Bach-y-Rita et al 2003). The emotional content of an image was rarely connected to a sensory substitution experience. However, qualia have been evident in some studies, in a limited fashion. For example, children were pleased and excited on perceiving for the first time the flickering flame of a candle after they blew on it (Miletic et al 1988). Data obtained here allow us to hypothesise that this was not the stimulation per se that fetched the affection but the particular spatial meaning assigned to it by subjects depending on the context. They viewed the upward triangle stimulation as a key, which allowed them to carry on their way.

The main results obtained by us offer prospects for studies leading to therapeutic, educational and game applications employing sensory substitution devices oriented towards visually impaired people. Our methodological approach could constitute a background for perceptual learning programmes aiming to give knowledge about visual-spatial concepts, particularly the ones relative to depth. Optimal learning conditions could be further established via performance-based criterion (ie the access to the next session being dependent on a fixed percentage of correct replies-Miletic 1994; Miletic et al 1988).

The navigation task used here, although realised in a maze via a radio-controlled mobile, is innovative in various aspects. Indirectly, it revealed the possibility of navigation through the environment thanks to a TVSS device. The real movement of people through space would associate exteroceptive and proprioceptive information. Thus, the navigation task described here could constitute a preliminary familiarisation step to the usage of TVSS devices in a larger real space. Moreover, our results allow us to consider the possibility of developing practical devices of visual substitution for very young blind subjects, helping to avoid 
developmental disorders related to early blindness. For example, when the attraction of the unusual stimulation produced by a TVSS was evaluated in babies (Segond et al 2007), two micro cameras (only one being active) were placed on the baby's feet. The active camera placed randomly on the left or right foot conveyed visual images into binary data transmitted to a matrix placed on the abdomen. A procedure of free-operant conditioning of the foot kicks was used. The TVSS stimulation was found very attractive. Babies learnt very quickly, since they do not need any training procedure (equipped with very potent cerebral plasticity), how to get and repeat the TVSS stimulation (by moving foot with active camera).

All the findings here are even more encouraging as they were obtained with a lowresolution 64-taxel TVSS device. Behavioural data obtained in our experiments give credence to further experiments on biological substrates of sensory substitution evaluated by neuro-imagery. In addition, not only the PTD version of the TVSS, constructed specially from nonmagnetic elements, but also the miniaturised electronic TDU version (matrix of $3 \mathrm{~cm} \times 3 \mathrm{~cm}$ with 144 electrodes explored by the tongue) can be safely used in the fMRI environment. This unlocks huge new prospects for scientific investigation.

Acknowledgments. This research was supported by the European Commission Quality of Life contract QLG-3CT-2000-01797, 'Brain Plasticity and Sensory Substitution in Human Blindness'. We gratefully thank Laurence de Lussy-Kubisa and Vincent Chrétiennot for their assistance and/or technical help, and the volunteers who gave of their time to participate in the study.

\section{References}

Arditi A, Holtzman J D, Kosslyn S M, 1988 "Mental imagery and sensory experience in congenital blindness" Neuropsychologia 26 1-12

Arno P, Capelle C, Wanet-Defalque M C, Catalan-Ahumada M, Veraart C, 1999 "Auditory coding of visual patterns for the blind" Perception 28 1013-1029

Arno P, Vanlierde A, Streel E, Wanet-Defalque M-C, Sanabria-Bohorquez S, Veraart C, 2001 "Auditory coding of visual patterns for the blind" Applied Cognitive Psychology 15 509-519

Auvray C, Hanneton S, Lenay C, O'Regan J K, 2005 "There is something out there: distal attribution in sensory substitution, twenty years later" Journal of Integrative Neuroscience 4 505-521

Bach-y-Rita P, 1972 Brain Mechanisms in Sensory Substitution (New York: Academic Press)

Bach-y-Rita P, 1988 "Brain plasticity", in Rehabilitation Medicine Ed. J Goodgold (St Louis, MO: C V Mosby Co) pp 113-118

Bach-y-Rita P, 1995 Nonsynaptic Diffusion Neurotransmission and Late Brain Reorganization (New York: Demos-Vermande)

Bach-y-Rita P, 1997 "Substitution sensorielle et qualia", in Perception et Intermodalité: Approches Actuelles de la Question de Molyneux Ed. J Proust (Paris: Presses Universitaires de France) pp 81-100

Bach-y-Rita P, Hughes B, 1985 "Tactile vision substitution: some instrumentation and perceptual considerations", in Electronic Spatial Sensing for the Blind Eds D H Warren, E R Strelow (Dordrecht: Martinus Nijhoff) pp 172-186

Bach-y-Rita P, Kaczmarek K A, Tyler M E, 2003 "A tongue-based tactile display for portrayal of environmental characteristics", in Psychological Issues in the Design and Use of Virtual and Adaptative Environments Eds L Hettlinger, M Haas (Mahwah, NJ: Lawrence Erlbaum Associates) pp 169-186

Collins C C, Bach-y-Rita P, 1970 "Transmission of pictorial information through the skin" Advances in Biological \& Medical Physics 14 285-315

Delorme A, 2003 “L'organisation perceptive", in Perception et Réalité. Une Introduction à la Psychologie des Perceptions Eds A Delorme, M Flückiger (Paris: De Boeck) pp 225-246

Gibson J J, 1950 The Perception of the Visual Field (Boston, MA: Houghton Mifflin)

Gibson J J, 1966 The Senses Considered as Perceptual Systems (Boston, MA: Houghton Mifflin)

Gibson J J, 1979 The Ecological Approach to Visual Perception (Boston, MA: Houghton Mifflin) 
Grant A C, Thiagarajah M C, Sathian K, 2000 "Tactile perception in blind Braille readers: A psychophysical study of acuity and hyperacuity using gratings and dot patterns" Perception \& Psychophysics 62 301-312

Guarniero G, 1974 "Experience of tactile vision" Perception 3 101-104

Guarniero G, 1977 “Tactile vision: A personal view” Journal of Visual Impairment \& Blindness 71 $125-130$

Hamilton R H, Pascual-Leone A, 1998 "Cortical plasticity associated with Braille learning” Trends in Cognitive Sciences 2 168-174

Hatwell Y, 2003 Psychologie Cognitive de la Cécité Précoce (Paris: Dunod)

Held R, Durlach N, 1992 "Telepresence" Presence 1 109-112

Heller M A, Brackett D D, Scroggs E, Steffen H, Heatherly K, Salik S, 2002 "Tangible pictures: Viewpoint effects and linear perspective in visually impaired people" Perception 31 747-769

Heller M A, Calcaterra J A, Tyler L A, Burson L L, 1996 "Production and interpretation of perspective drawings by blind and sighted people" Perception 25 321-334

Jansson G, 1983 "Tactile guidance of movement" International Journal of Neuroscience 19 37-45

Kaczmarek K, Bach-y-Rita P, 1995 "Tactile displays", in Virtual Environments and Advanced Interface Design Eds W Barfield, T A Furness (Oxford: Oxford University Press) pp 349-414

Lenay C, Gapenne O, Hanneton S, Marque C, Genouel C, 2000 "La substitution sensorielle: limites et perspectives", in Toucher pour Connaître. Psychologie Cognitive de la Perception Tactile Manuelle Eds Y Hatwell, A Streri (Paris: Presses Universitaires de France) pp 287-306. Published in English as Touch for Knowing (Amsterdam: John Benjamins, 2003)

McLinden D J, 1988 "Spatial task performance: A meta-analysis" Journal of Visual Impairment \& Blindness 82 231-236

Maguire E, Burgess N, O'Keefe J, 1999 “Human spatial navigation: cognitive maps, sexual dimorphism and neural substrates" Current Opinion in Neurobiology 9 171-177

Miletic G, 1994 "Vibrotactile perception: Perspective taking by children who are visually impaired" Journal of Visual Impairment \& Blindness 88 550-563

Miletic G, Hughes B, Bach-y-Rita P, 1988 "Vibrotactile stimulation: An educational program for spatial concept development" Journal of Visual Impairment \& Blindness 82 366-370

Proust J, 1997 Perception et Intermodalité: Approches Actuelles de la Question de Molyneux (Paris : Presses Universitaires de France)

Sampaio E, 1989 "L'autisme infantile: le cas de l'enfant aveugle. Réflexions méthodologiques" Psychologie Médicale $212020-2024$

Sampaio E, 1995 "Les substitutions sensorielles adaptées aux déficits visuels importants", in Le Déficit Visuel. Des Fondements Neuropsychologiques à la Pratique de la Réadaptation Eds A B Safran, A Assimacopoulos (Paris: Masson) pp 197-211

Sampaio E, Maris S, Bach-y-Rita P, 2001 "Brain plasticity: 'visual' acuity of blind persons via the tongue" Brain Research 908 204-207

Segond, H, 2008 "Le toucher en développement: Perception tactile et continuité transnatale", in Cognition, Santé et Vie Quotidienne volume 1, Ed. G Chasseigne (Reims: Publibook Université) pp 75-108

Segond H, Weiss D, Sampaio E, 2005 "Human spatial navigation via a visuo-tactile sensory substitution system" Perception 34 1231-1249

Segond H, Weiss D, Sampaio E, 2007 "A proposed tactile-stimulation system for infants who are blind tested on sighted infants" Journal of Visual Impairment \& Blindness 101 32-43

Streri A, Gentaz E, 2003 "Cross modal recognition of shape from hand to eye in human newborns" Somatosensory and Motor Research 20 11-16

Van Horn J D, Gold J M, Esposito G, Ostrem J L, Mattay V, Weinberger D R, Berman K F, 1998 "Changing patterns of brain activation during maze learning" Brain Research 793 29-38

Zappe A C, Maucher T, Meier K, Scheiber C, 2004 "Evaluation of a pneumatically driven tactile stimulator device for vision substitution during fMRI studies" Magnetic Resonance in Medicine $51828-834$ 